\title{
Insect ventral radar cross-section polarization dependence measurements for radar entomology
}

\author{
S.E. $\operatorname{Hobbs}^{(a)}$ \\ A.C. Aldhous ${ }^{(b)}$ \\ (a) School of Engineering, Cranfield University \\ Cranfield, Bedford, MK43 0AL, UK \\ (b) CAD Schroer UK Ltd, 39 Newnham Road, \\ Cambridge, CB3 9EY, UK
}

19 June 2006

\begin{abstract}
Radar entomology has developed such that routine long-term monitoring of insect flight through the atmospheric boundary layer is now practical. Typical entomological radars use X-band $(9.4 \mathrm{GHz})$ marine transceivers with a vertical pencil beam and rotate the plane of polarization about the beam axis. Ideally, insect species and other parameters (mass, etc.) should be estimated from the measured radar cross-section variation with polarization angle. For this, a library of known insect crosssection polarization signatures is required. Two models are currently used to parameterize the polarization signature: the harmonic model and a model using the scattering matrix for symmetric targets (SM3).

Data from the literature and a doctoral research project are presented and analysed to obtain parameters for both the harmonic and SM3 models. Knowledge of the measurement errors allows SM3 parameter uncertainties to be quantified in most cases using a maximum likelihood approach.

Results for 68 insects representing 24 species are presented. These include several economically significant species (e.g. bees and locusts), with individual insect masses ranging from $9 \mathrm{mg}$ to $3 \mathrm{~g}$.
\end{abstract}

\section{Introduction}

Radar entomology has become a practical long-term monitoring technique in the last decade [1, 2, 3] after gradual development since the 1970's. Radar's ability to monitor insect flight remotely is practically unique and provides valuable insights into insect behaviour, e.g. [4]. Current operational systems use a vertically pointing linearly polarized beam and marine X-band transceivers, and rotate the plane of polarization about the vertical axis at several Hz. The beam is slightly offset from the vertical axis (by $10 \%$ of the beamwidth typically) and scanned around the vertical at the same rate. The combination of modulations due to the beam motion and polarization rotation are used to measure the target's trajectory and radar cross-section (RCS) parameters (e.g. $[5,6,7])$. 
It is highly desirable to be able to identify insect species and estimate significant parameters such as mass from the RCS data. So far, only a few sets of measurements of insect RCS and their physical data (species, mass, body dimensions, etc.) have been widely available [8, 9]. This article reports measurements from the experiments of Aldhous [10], and presents them and the data of [9] in formats compatible with current operational systems. Specifically, the measurements are of the polarization dependence of the insects' ventral RCS, i.e. directly comparable with the measurements from typical operational entomological radars.

The following sections describe parameterizations of the polarization dependence of insect RCS, the method used to measure insect RCS, the results and the insects' physical data, and a brief discussion.

\section{Parameterizations of RCS Polarization De- pendence}

The scattering matrix $(S)$ provides a complete description of the RCS linear polarization dependence for a fixed target aspect. The RCS $(\sigma)$ is obtained by pre- and post-multiplying $S$ by vectors corresponding to the received and transmitted polarizations:

$$
\sigma=\left|\mathbf{h}_{r}^{* T} S \mathbf{h}_{t}\right|^{2}
$$

The general form of $S$ is

$$
S=e^{i \phi}\left(\begin{array}{ll}
\sqrt{\sigma_{x x}} & \sqrt{\sigma_{x y}} e^{i \alpha} \\
\sqrt{\sigma_{y x}} e^{i \alpha^{\prime}} & \sqrt{\sigma_{y y}} e^{i \beta}
\end{array}\right)
$$

This can be simplified significantly in practice to obtain the models used currently. First, the phase factor $\phi$ corresponds to range and is usually ignored. Second, since scattering from insects is linear, $S$ must be symmetrical. A third simplification comes if the $x$ or $y$ axis lies in a plane of symmetry of the target since then the off-diagonal terms are zero. Applying the first two simplifications, equation 1 can be expanded and by grouping coefficients of the $2 \theta$ and $4 \theta$ terms leads to the "harmonic" form of the RCS polarization dependence equation as used by Aldhous [10]:

$$
\sigma=a_{0}+a_{1} \cos 2\left(\theta-\theta_{1}\right)+a_{2} \cos 4\left(\theta-\theta_{2}\right)
$$

This is general and has become widely used. The $a_{i}$ and $\theta_{i}$ can be derived from the components of $S$. A weakness of the harmonic model is that physically unrealistic values are possible for the coefficients $a_{i}$ and care is needed to avoid these [7].

Applying the third simplification to $S$ leads to a model which assumes symmetry, makes orientation (the rotation used to align axes with a symmetry plane) explicit, and ensures physical plausibility. This model is labelled "SM3", because of the three scattering matrix parameters used $\left(\epsilon^{2}=\sigma_{y y} / \sigma_{x x}\right)$ :

$$
\sigma=\sigma_{x x}\left|\left(\cos \left(\theta-\theta_{0}\right), \sin \left(\theta-\theta_{0}\right)\right)\left(\begin{array}{ll}
1 & 0 \\
0 & \epsilon e^{i \beta}
\end{array}\right)\left(\begin{array}{c}
\cos \left(\theta-\theta_{0}\right) \\
\sin \left(\theta-\theta_{0}\right)
\end{array}\right)\right|^{2}
$$


These models of polarization dependence allow the full variation to be captured with only $3-5$ parameters. The harmonic model has five parameters, of which one $\left(\theta_{1}\right)$ is taken as the insect orientation. The SM3 model uses four parameters: $\theta_{0}$ is identified explicitly as the orientation of the symmetry plane and the other three $\left(\sigma_{x x}, \epsilon, \beta\right)$ define the target's RCS. The harmonic model parameters can be derived from the scattering matrix, and the SM3 model parameters can be obtained from either the scattering matrix or the harmonic model. For both models "orientation" can only be measured with an ambiguity of $180^{\circ}$. The peak signal may be aligned with or perpendicular to the insect's body axis depending on its size relative to the wavelength [8].

\section{Insect RCS Measurements and Data Analysis}

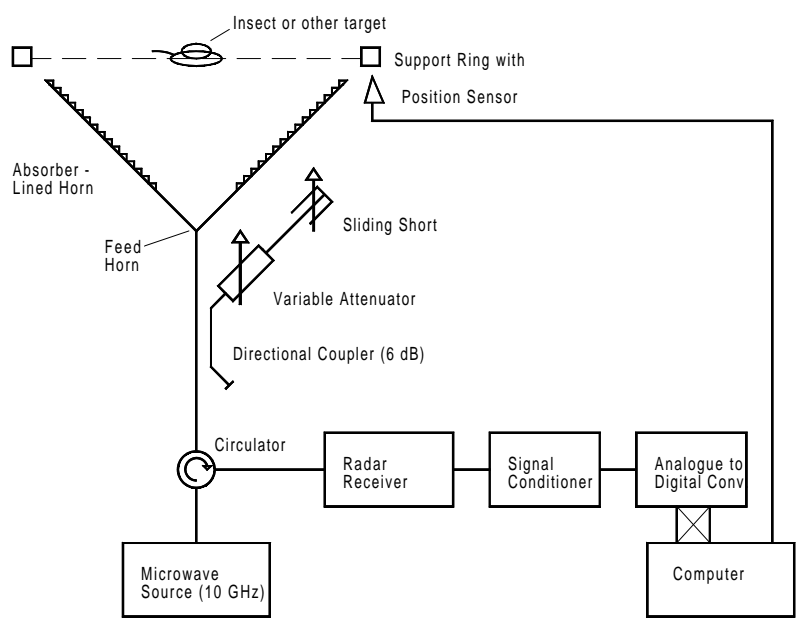

Figure 1: Experiment test rig.

Figure 1 shows the main equipment used. The CW X-band microwave source provides power to illuminate the target. A small fraction of the reflected power is collected by the feed-horn, and then separated from the outgoing power by the circulator and directed to the receiver. The directional coupler / attenuator / sliding short assembly is adjusted to provide a small reflected signal exactly in antiphase with any unwanted cross-coupling or reflections due to impedance mismatches or side-lobes. Null adjustment is delicate and typically remained stable for several minutes. The microwave parts and horn were contained in a box about $1.5 \mathrm{~m}$ square to protect them from draughts (and so ensure temperature stability). The rig was used outdoors in dry, calm weather with the horn aperture plane horizontal (the antenna beam was vertical upwards). This minimized unwanted reflections from objects other than the insect target being measured. The range from the feed horn to the target was $0.75 \mathrm{~m}$, which is far enough (beyond the near-field) to ensure that the target receives plane wave illumination. The target, supported using thin nylon line close to the beam axis, was rotated about a vertical axis with the beam fixed. Care was taken to use 
live insect targets, anaesthetized using carbon dioxide gas to prevent movement during measurements.

A measurement cycle includes receiver nulling (no target), calibration (using a reference target), and measurement of the insect backscatter, followed by a final null check. Only data with no significant null drift were accepted. The measurements taken comprise 256 reflected power measurements for every $180^{\circ}$ rotation of the insect, repeated for 10 full rotations. The data stored were equivalent to mean and standard deviation of the backscattered power for the 20 samples taken at each of the 256 angle steps in $180^{\circ}$. Full details of the experimental method are given in [10].

\subsection{Data Analysis}

The data recorded after application of the receiver calibration consist of insect radar cross-section measurements as a function of polarization angle. From these, a least squares method was used to estimate the harmonic model parameters: these are recorded in Table 2 . Table 1 gives the key physical data for the insects (species, mass, and dimensions).

The SM3 model parameters were estimated from the harmonic parameters by a maximum-likelihood (Levenberg-Marquardt [11]) method. A numerical method was used instead of an analytical solution because the maximumlikelihood approach is inherently suited to quantitative parameter uncertainty estimatation, and the algorithm could also be used directly on experimental data. Since the original data are no longer available, synthetic measurements were created using the harmonic model parameters. Measurement uncertainty was obtained from actual measurements for insects 22 and 26 of Tables 1 to 3. For all other insects, the noise model (equation 6 below) was used (for RCS above $3.5 \mathrm{~cm}^{2}$ the model had to be extrapolated beyond the calibration data). Care was taken to account correctly for the number of degrees of freedom when fitting the SM3 model to the synthesised data. Since no measurement uncertainty information is available in [9], a standard least squares solution method is used for these data and no parameter uncertainties are reported.

\subsection{Quantifying Measurement Noise}

To assess the accuracy of fitted parameters it is necessary to have a model of the measurement noise. [10, Figures 4.3, 5.5] gives measurement uncertainties expressed as a fraction of the ADC output for two insect targets (insects 22 and 26 of Table 1). These fractional measurement standard deviations $(\delta f=\delta n / n)$ are converted into equivalent RCS standard deviation $(\delta \sigma)$ using the receiver calibration. The calibration is expressed as $z=F(n)\left(n=F^{-1}(z)\right.$ is its inverse) using $z=\log _{10}\left(\sigma / \mathrm{cm}^{2}\right)$ where $n$ is the ADC output digital number. $F^{-1}(z)$ and its derivative are evaluated numerically using calibration data.

$$
\begin{aligned}
\delta \sigma & =\frac{d \sigma}{d n} \delta n=\frac{d \sigma}{d n} \cdot n \cdot \delta f \\
& =\frac{1}{\frac{d n}{d \sigma}} \cdot n \cdot \delta f=\frac{1}{\left(\frac{d n}{d z} \frac{d z}{d \sigma}\right)} \cdot n \cdot \delta f
\end{aligned}
$$




$$
=\frac{\sigma \ln 10}{\frac{d F^{-1}(z)}{d z}} \cdot F^{-1}(z) \cdot \delta f
$$

Equation 5 was used to obtain measurement uncertainties (see Figure 2) for insects 22 and 26 of [10]. From these a general measurement uncertainty model (equation 6) has been developed. The model is conservative and therefore tends to overestimate the errors.

$$
\delta \sigma=0.040 \mathrm{~cm}^{2}+0.068 \sigma
$$

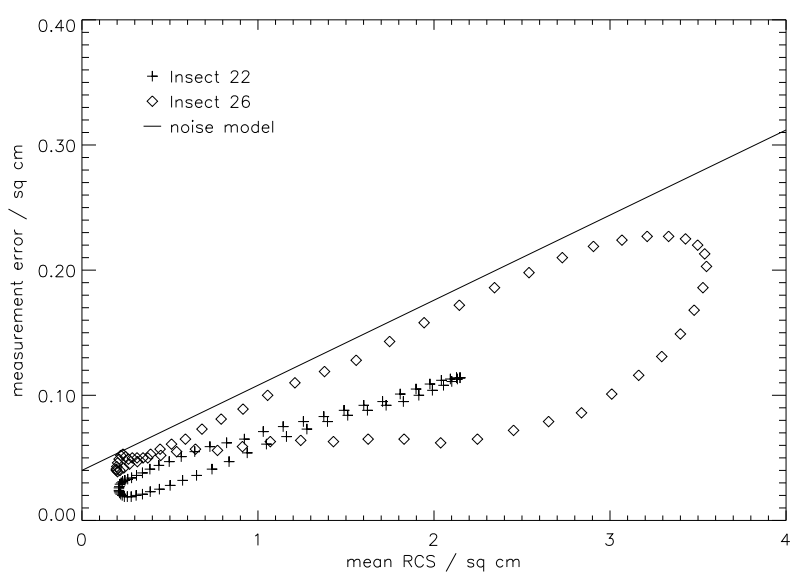

Figure 2: Measurement noise standard deviation as a function of mean RCS for the insects of [10].

\section{Results}

Tables 1 and 2 list the insect data from [10] to which the SM3 model parameters have been fitted. Tables 4 and 5 list the source data used from [9]. The results of the SM3 model fitting are given in Tables 3 and 5. As discussed above, SM3 parameter uncertainties (one standard deviation values) are only stated for the data of [10], and then only when the fit of the SM3 model to the harmonic model is satisfactory. For insects 46-48, the degree of asymmetry in the reported RCS is too great for the SM3 model to give a good fit, and so no uncertainties are stated.

Attempts have been made to relate the physical data to the RCS parameters using both formats. No simple, comprehensive relationships have yet been found although approximate mass estimates are possible (see [10, 12]). The difficulty in interpreting the RCS parameters for these insects stems from the fact that their scattering at X-band $(9.4 \mathrm{GHz})$ is generally in the Mie region. [12] presents the following equations suitable for estimating insect mass $(m)$ from RCS parameters $\left(\sigma_{x x}, a_{0}\right)$ for different mass ranges. These empirical relationships are generally accurate to within a factor of 2 for insect masses from $\mathrm{mg}$ to g. 


$$
\begin{aligned}
& m / \mathrm{mg}=39.5 \sqrt{\sigma_{x x} / \mathrm{cm}^{2}} \quad \begin{array}{l}
\text { For } \sigma_{x x} \leq 0.0032 \mathrm{~cm}^{2} \\
m / \mathrm{mg}=39.5 \sqrt{a_{0} / \mathrm{cm}^{2}} \quad \text { For } \sigma_{x x}>0.0032 \mathrm{~cm}^{2} \text { and } a_{0}<0.25 \mathrm{~cm}^{2} \\
\log _{10}(m / \mathrm{mg})=\begin{array}{r}
2.205+0.8729 \log _{10}\left(a_{0} / \mathrm{cm}^{2}\right) \\
+0.3323 \log _{10}^{2}\left(a_{0} / \mathrm{cm}^{2}\right)
\end{array} \quad \text { For } a_{0} \geq 0.25 \mathrm{~cm}^{2}
\end{array}
\end{aligned}
$$

\begin{tabular}{|c|c|c|c|c|c|c|}
\hline \multicolumn{3}{|c|}{ Insect number, symbol, and species } & \multirow{2}{*}{$\begin{array}{r}\text { Mass } \\
\text { mg }\end{array}$} & \multirow{2}{*}{$\begin{array}{r}\text { Wing } \\
\text { span } \\
\text { mm }\end{array}$} & \multirow{2}{*}{$\begin{array}{r}\text { Body } \\
\text { length } \\
\mathrm{mm}\end{array}$} & \multirow{2}{*}{$\begin{array}{r}\text { Abdomen } \\
\text { width } \\
\text { mm } \\
\end{array}$} \\
\hline & & & & & & \\
\hline 1 & $\mathrm{~A}$ & Tipula oleracea Linnaeus & 45 & 14.8 & 17.7 & 1.7 \\
\hline 2 & $\mathrm{~A}$ & Tipula oleracea L. & 49 & 17.2 & 18.7 & 1.6 \\
\hline 3 & $\mathrm{~B}$ & Alcis rependata rependata $\mathrm{L}$. & 52 & 20.2 & 18.9 & 2.9 \\
\hline 4 & $\mathrm{C}$ & Mesapamea secalis $\mathrm{L}$. & 53 & 14.5 & 14.0 & 3.0 \\
\hline 5 & $\mathrm{D}$ & Chorthippus brunneus Thunberg & 68 & 13.8 & 15.6 & 2.1 \\
\hline 6 & $\mathrm{D}$ & Chorthippus brunneus T. & 97 & 13.7 & 16.9 & 1.9 \\
\hline 7 & $\mathrm{D}$ & Chorthippus brunneus T. & 173 & 11.5 & 20.2 & 2.9 \\
\hline 8 & $\mathrm{D}$ & Chorthippus brunneus T. & 188 & 11.7 & 22.0 & 2.9 \\
\hline 9 & $\mathrm{D}$ & Chorthippus brunneus T. & 190 & 12.0 & 20.8 & 3.0 \\
\hline 10 & $\mathrm{D}$ & Chorthippus brunneus T. & 200 & 12.4 & 21.3 & 3.0 \\
\hline 11 & $\mathrm{D}$ & Chorthippus brunneus T. & 214 & 15.4 & 21.7 & 2.9 \\
\hline 12 & $\mathrm{E}$ & Noctua janthina Denis \& Schiffermüller & 80 & 16.5 & 15.5 & 4.5 \\
\hline 13 & $\mathrm{E}$ & Noctua janthina D\&S & 82 & 17.0 & 17.0 & 4.0 \\
\hline 14 & $\mathrm{~F}$ & Hepialus sylvina $\mathrm{L}$. & 82 & 14.1 & 15.5 & 3.0 \\
\hline 15 & $\mathrm{~F}$ & Hepialus sylvina L. & 113 & 15.4 & 17.3 & 3.5 \\
\hline 16 & $\mathrm{G}$ & Ochropleura plecta L. & 100 & 13.2 & 14.2 & 4.1 \\
\hline 17 & $\mathrm{H}$ & Xestia xanthographa D\&S & 102 & 16.8 & 16.3 & 3.2 \\
\hline 18 & $\mathrm{H}$ & Xestia xanthographa D\&S & 126 & 16.9 & 17.8 & 4.2 \\
\hline 19 & I & Autographa gamma L. & 107 & 20.0 & 20.5 & 4.4 \\
\hline 20 & $\mathrm{I}$ & Autographa gamma L. & 118 & 20.2 & 19.4 & 4.4 \\
\hline 21 & $\mathrm{I}$ & Autographa gamma L. & 149 & 20.6 & 22.9 & 4.7 \\
\hline 22 & $\mathrm{~J}$ & Amphipyra tragopyinis Clerck & 110 & 18.4 & 17.9 & 4.3 \\
\hline 23 & $\mathrm{~K}$ & Xestia c-nigrum L. & 125 & 17.7 & 17.5 & 5.0 \\
\hline 24 & $\mathrm{~L}$ & Aglais urticae L. & 128 & 25.4 & 21.5 & 4.2 \\
\hline 25 & $\mathrm{~L}$ & Aglais urticae L. & 160 & 25.8 & 21.2 & 3.6 \\
\hline 26 & M & Noctua comes Hübner & 133 & 17.5 & 18.5 & 4.5 \\
\hline 27 & M & Noctua comes $\mathrm{H}$. & 176 & 18.6 & 19.7 & 4.9 \\
\hline 28 & $\mathrm{~N}$ & Agrotis exclamationis L. & 208 & 17.5 & 19.0 & 5.5 \\
\hline 29 & $\mathrm{O}$ & Noctua pronuba L. & 270 & 25.4 & 27.3 & 6.6 \\
\hline 30 & $\mathrm{O}$ & Noctua pronuba L. & 295 & 23.5 & 23.0 & 5.7 \\
\hline 31 & $\mathrm{O}$ & Noctua pronuba L. & 321 & 25.0 & 26.0 & 5.0 \\
\hline 32 & $\mathrm{O}$ & Noctua pronuba L. & 333 & 24.5 & 24.5 & 7.0 \\
\hline 33 & $\mathrm{O}$ & Noctua pronuba L. & 337 & 25.5 & 25.0 & 6.5 \\
\hline 34 & $\mathrm{O}$ & Noctua pronuba L. & 400 & 26.5 & 26.8 & 6.2 \\
\hline 35 & $\mathrm{O}$ & Noctua pronuba L. & 419 & 25.3 & 25.8 & 7.1 \\
\hline
\end{tabular}

Table 1: Physical data and species of measured insects [10]. 
continued from previous page

\begin{tabular}{|c|c|c|c|c|c|c|}
\hline \multicolumn{3}{|c|}{ Insect number, symbol, and species } & \multirow{2}{*}{$\begin{array}{r}\text { Mass } \\
\text { mg }\end{array}$} & \multirow{2}{*}{$\begin{array}{r}\text { Wing } \\
\text { span } \\
\text { mm }\end{array}$} & \multirow{2}{*}{$\begin{array}{r}\text { Body } \\
\text { length } \\
\mathrm{mm} \\
\end{array}$} & \multirow{2}{*}{$\begin{array}{r}\text { Abdomen } \\
\text { width } \\
\text { mm } \\
\end{array}$} \\
\hline & & & & & & \\
\hline 36 & $\mathrm{O}$ & Noctua pronuba L. & 443 & 27.7 & 26.9 & 6.7 \\
\hline 37 & $\mathrm{O}$ & Noctua pronuba L. & 451 & 24.5 & 26.0 & 7.5 \\
\hline 38 & $\mathrm{O}$ & Noctua pronuba L. & 457 & 26.1 & 27.0 & 7.6 \\
\hline 39 & $\mathrm{O}$ & Noctua pronuba L. & 459 & 26.1 & 25.5 & 7.0 \\
\hline 40 & $\mathrm{O}$ & Noctua pronuba L. & 495 & 25.0 & 28.8 & 7.5 \\
\hline 41 & $\mathrm{O}$ & Noctua pronuba L. & 538 & 26.9 & 27.5 & 7.8 \\
\hline 42 & $\mathrm{P}$ & Danaus plexippus L. & 305 & 39.5 & 30.2 & 3.7 \\
\hline 43 & Q & Noctua fimbriata Schreber & 648 & 24.5 & 24.5 & 9.0 \\
\hline 44 & $\mathrm{R}$ & Schistocerca gregaria Forsk & 1084 & 45.7 & 46.6 & 4.8 \\
\hline 45 & $\mathrm{R}$ & Schistocerca gregaria F. & 1133 & 48.9 & 50.4 & 5.6 \\
\hline 46 & $\mathrm{R}$ & Schistocerca gregaria F. & 1371 & 45.2 & 48.3 & 5.4 \\
\hline 47 & $\mathrm{R}$ & Schistocerca gregaria F. & 1494 & 45.3 & 47.5 & 5.1 \\
\hline 48 & $\mathrm{R}$ & Schistocerca gregaria F. & 1532 & 46.6 & 50.7 & 5.0 \\
\hline 49 & $\mathrm{R}$ & Schistocerca gregaria F. & 1713 & 56.1 & 54.5 & 6.5 \\
\hline 50 & $\mathrm{R}$ & Schistocerca gregaria F. & 1751 & 47.0 & 51.2 & 5.4 \\
\hline 51 & $\mathrm{R}$ & Schistocerca gregaria F. & 2241 & 51.9 & 56.7 & 6.1 \\
\hline 52 & $\mathrm{R}$ & Schistocerca gregaria F. & 2326 & 54.7 & 55.4 & 6.8 \\
\hline 53 & $\mathrm{R}$ & Schistocerca gregaria F. & 2474 & 53.3 & 58.1 & 6.6 \\
\hline 54 & $\mathrm{R}$ & Schistocerca gregaria F. & 3094 & 50.3 & 62.3 & 6.9 \\
\hline
\end{tabular}

Table 2: Harmonic RCS parameters for measured insects [10].

\begin{tabular}{rrrrrrr}
$\begin{array}{r}\text { Insect } \\
\text { number }\end{array}$ & $\begin{array}{r}\text { Species } \\
\text { symbol }\end{array}$ & $\begin{array}{r}a_{0} \\
\mathrm{~cm}^{2}\end{array}$ & $\begin{array}{r}a_{1} \\
\mathrm{~cm}^{2}\end{array}$ & $\begin{array}{r}a_{2} \\
\mathrm{~cm}^{2}\end{array}$ & $\begin{array}{r}\theta_{1} \\
{ }^{2}\end{array}$ & $\begin{array}{r}\theta_{2}-\theta_{1} \\
\circ\end{array}$ \\
\hline 1 & $\mathrm{~A}$ & 0.250 & 0.250 & 0.028 & -21.9 & 4.2 \\
2 & $\mathrm{~A}$ & 0.180 & 0.230 & 0.049 & -2.4 & 1.0 \\
3 & $\mathrm{~B}$ & 0.280 & 0.240 & 0.036 & -26.1 & -1.4 \\
4 & $\mathrm{C}$ & 0.091 & 0.091 & 0.002 & 10.0 & -13.4 \\
5 & $\mathrm{D}$ & 0.630 & 0.560 & 0.130 & -14.3 & -4.2 \\
6 & $\mathrm{D}$ & 0.390 & 0.440 & 0.100 & -4.8 & -3.6 \\
7 & $\mathrm{D}$ & 2.360 & 3.080 & 0.850 & 5.1 & -0.2 \\
8 & $\mathrm{D}$ & 1.730 & 2.260 & 0.740 & 0.1 & 0.4 \\
9 & $\mathrm{D}$ & 2.070 & 2.630 & 0.840 & 3.3 & 0.0 \\
10 & $\mathrm{D}$ & 1.570 & 2.000 & 0.870 & -2.6 & 1.3 \\
11 & $\mathrm{D}$ & 1.540 & 1.950 & 0.730 & 4.9 & 0.2 \\
12 & $\mathrm{E}$ & 0.730 & 0.590 & 0.078 & -2.7 & -4.0 \\
13 & $\mathrm{E}$ & 0.330 & 0.200 & 0.053 & 6.3 & 0.5 \\
14 & $\mathrm{~F}$ & 0.140 & 0.130 & 0.029 & 2.0 & 2.8 \\
15 & $\mathrm{~F}$ & 0.280 & 0.250 & 0.054 & 4.4 & 0.9 \\
16 & $\mathrm{G}$ & 0.700 & 0.660 & 0.140 & 1.5 & -0.2 \\
17 & $\mathrm{H}$ & 0.830 & 0.820 & 0.150 & -3.5 & -2.1 \\
18 & $\mathrm{H}$ & 1.030 & 1.160 & 0.290 & -0.3 & 0.8 \\
19 & $\mathrm{I}$ & 0.490 & 0.520 & 0.130 & 5.1 & -0.2 \\
20 & $\mathrm{I}$ & 0.880 & 0.820 & 0.180 & -1.5 & 1.5 \\
\hline & & & \multicolumn{5}{c}{ continued on next page }
\end{tabular}




\begin{tabular}{|c|c|c|c|c|c|c|}
\hline $\begin{array}{r}\text { Insect } \\
\text { number }\end{array}$ & $\begin{array}{l}\text { Species } \\
\text { symbol }\end{array}$ & $\begin{array}{r}a_{0} \\
\mathrm{~cm}^{2}\end{array}$ & $\begin{array}{r}a_{1} \\
\mathrm{~cm}^{2}\end{array}$ & $\begin{array}{r}a_{2} \\
\mathrm{~cm}^{2}\end{array}$ & $\begin{array}{r}\theta_{1} \\
\circ\end{array}$ & $\theta_{2}-\theta_{1}$ \\
\hline 21 & I & 1.500 & 1.580 & 0.310 & -6.6 & -0.7 \\
\hline 22 & $\mathrm{~J}$ & 0.980 & 0.970 & 0.200 & -1.6 & -0.2 \\
\hline 23 & $\mathrm{~K}$ & 1.200 & 1.360 & 0.340 & -5.0 & 0.4 \\
\hline 24 & $\mathrm{~L}$ & 1.260 & 1.310 & 0.310 & 8.2 & 0.3 \\
\hline 25 & $\mathrm{~L}$ & 1.220 & 1.110 & 0.210 & -13.5 & 5.8 \\
\hline 26 & M & 1.510 & 1.670 & 0.370 & 1.2 & -1.6 \\
\hline 27 & M & 1.620 & 1.750 & 0.380 & -2.3 & -0.6 \\
\hline 28 & $\mathrm{~N}$ & 1.850 & 1.360 & 0.250 & 0.7 & -2.1 \\
\hline 29 & $\mathrm{O}$ & 1.840 & 1.540 & 0.310 & 0.4 & -0.6 \\
\hline 30 & $\mathrm{O}$ & 1.660 & 1.350 & 0.410 & -3.8 & 0.4 \\
\hline 31 & $\mathrm{O}$ & 0.970 & 0.870 & 0.310 & 3.5 & 1.0 \\
\hline 32 & $\mathrm{O}$ & 2.720 & 2.030 & 0.140 & -3.6 & -8.3 \\
\hline 33 & $\mathrm{O}$ & 1.590 & 0.850 & 0.380 & -4.8 & 2.3 \\
\hline 34 & $\mathrm{O}$ & 1.880 & 1.740 & 0.280 & -0.5 & -2.0 \\
\hline 35 & $\mathrm{O}$ & 1.840 & 0.700 & 0.430 & -1.6 & 0.9 \\
\hline 36 & $\mathrm{O}$ & 2.330 & 0.770 & 0.500 & -3.1 & 2.0 \\
\hline 37 & $\mathrm{O}$ & 2.280 & 0.290 & 0.520 & -1.8 & 1.1 \\
\hline 38 & $\mathrm{O}$ & 2.710 & 0.360 & 0.620 & -11.6 & 10.4 \\
\hline 39 & $\mathrm{O}$ & 1.770 & 0.980 & 0.620 & -8.7 & 2.1 \\
\hline 40 & $\mathrm{O}$ & 2.550 & 0.710 & 0.550 & 4.0 & 0.9 \\
\hline 41 & $\mathrm{O}$ & 2.490 & 0.190 & 0.550 & -46.6 & -1.1 \\
\hline 42 & $\mathrm{P}$ & 2.210 & 1.960 & 0.370 & 4.9 & -0.7 \\
\hline 43 & Q & 2.160 & 0.220 & 0.360 & -89.4 & -1.2 \\
\hline 44 & $\mathrm{R}$ & 4.110 & 2.220 & 1.170 & -86.8 & -1.8 \\
\hline 45 & $\mathrm{R}$ & 4.580 & 1.600 & 0.830 & -80.8 & -5.7 \\
\hline 46 & $\mathrm{R}$ & 4.690 & 1.670 & 1.170 & -75.3 & -11.5 \\
\hline 47 & $\mathrm{R}$ & 5.300 & 2.550 & 1.220 & -78.5 & -7.2 \\
\hline 48 & $\mathrm{R}$ & 4.880 & 0.880 & 0.600 & -60.2 & -20.1 \\
\hline 49 & $\mathrm{R}$ & 9.240 & 2.050 & 0.370 & -75.9 & -12.8 \\
\hline 50 & $\mathrm{R}$ & 5.440 & 4.150 & 1.320 & -83.7 & -2.4 \\
\hline 51 & $\mathrm{R}$ & 9.640 & 4.610 & 0.970 & -89.5 & -2.0 \\
\hline 52 & $\mathrm{R}$ & 10.900 & 4.960 & 1.340 & -78.1 & -7.1 \\
\hline 53 & $\mathrm{R}$ & 7.170 & 6.420 & 1.760 & 88.3 & -2.2 \\
\hline 54 & $\mathrm{R}$ & 16.800 & 12.500 & 3.210 & -89.0 & -2.1 \\
\hline
\end{tabular}

Table 3: SM3 RCS parameters for measured insects of [10]. For each parameter (e.g. $\sigma_{x x}$ ) the one standard deviation uncertainty (e.g. $\delta \sigma_{x x}$ ) is stated if the model fit is satisfactory.

\begin{tabular}{rrrrrrrrrr}
$\begin{array}{r}\text { Insect } \\
\text { number }\end{array}$ & $\begin{array}{r}\text { Species } \\
\text { symbol }\end{array}$ & $\begin{array}{r}\sigma_{x x} \\
\mathrm{~cm}^{2}\end{array}$ & $\begin{array}{r}\delta \sigma_{x x} \\
\mathrm{~cm}^{2}\end{array}$ & $\epsilon$ & $\delta \epsilon$ & $\begin{array}{r}\beta \\
\circ\end{array}$ & $\begin{array}{r}\delta \beta \\
\circ\end{array}$ & $\begin{array}{r}\theta_{0} \\
\circ\end{array}$ & $\begin{array}{r}\delta \theta_{0} \\
\circ\end{array}$ \\
\hline 1 & $\mathrm{~A}$ & 0.537 & 0.009 & 0.264 & 0.0133 & 0.0 & 14.80 & -21.9 & 0.57 \\
2 & $\mathrm{~A}$ & 0.460 & 0.009 & 0.062 & 0.0218 & 0.0 & 47.10 & -2.3 & 0.56 \\
3 & $\mathrm{~B}$ & 0.556 & 0.010 & 0.369 & 0.0157 & 32.9 & 10.90 & -26.1 & 0.62 \\
4 & $\mathrm{C}$ & 0.193 & 0.006 & 0.281 & 0.0315 & 0.0 & 19.30 & 9.9 & 1.30 \\
\hline
\end{tabular}




\begin{tabular}{|c|c|c|c|c|c|c|c|c|c|}
\hline $\begin{array}{r}\text { Insect } \\
\text { number }\end{array}$ & $\begin{array}{l}\text { Species } \\
\text { symbol }\end{array}$ & $\begin{array}{r}\sigma_{x x} \\
\mathrm{~cm}^{2}\end{array}$ & $\begin{array}{r}\delta \sigma_{x x} \\
\mathrm{~cm}^{2}\end{array}$ & $\epsilon$ & $\delta \epsilon$ & $\begin{array}{c}\beta \\
\beta \\
\circ\end{array}$ & $\begin{array}{r}\delta \beta \\
\circ\end{array}$ & $\begin{array}{r}\theta_{0} \\
\circ\end{array}$ & $\begin{array}{r}\delta \theta_{0} \\
\circ\end{array}$ \\
\hline 5 & $\mathrm{D}$ & 1.314 & 0.016 & 0.383 & 0.0077 & 59.7 & 3.22 & -14.4 & 0.36 \\
\hline 6 & $\mathrm{D}$ & 0.927 & 0.013 & 0.223 & 0.0152 & 61.6 & 6.70 & -4.9 & 0.37 \\
\hline 7 & $\mathrm{D}$ & 6.290 & 0.048 & 0.144 & 0.0042 & 102.1 & 1.79 & 5.2 & 0.14 \\
\hline 8 & $\mathrm{D}$ & 4.730 & 0.038 & 0.210 & 0.0039 & 119.5 & 1.39 & 0.1 & 0.15 \\
\hline 9 & $\mathrm{D}$ & 5.540 & 0.043 & 0.225 & 0.0034 & 111.2 & 1.25 & 3.3 & 0.14 \\
\hline 10 & $\mathrm{D}$ & 4.452 & 0.035 & 0.311 & 0.0033 & 139.5 & 1.11 & -2.2 & 0.14 \\
\hline 11 & $\mathrm{D}$ & 4.220 & 0.034 & 0.275 & 0.0037 & 124.0 & 1.18 & 4.9 & 0.15 \\
\hline 12 & $\mathrm{E}$ & 1.394 & 0.017 & 0.392 & 0.0074 & 22.3 & 8.38 & -2.5 & 0.38 \\
\hline 13 & $\mathrm{E}$ & 0.583 & 0.010 & 0.560 & 0.0115 & 58.4 & 3.98 & 6.4 & 0.74 \\
\hline 14 & $\mathrm{~F}$ & 0.299 & 0.008 & 0.359 & 0.0278 & 59.4 & 10.10 & 2.3 & 0.92 \\
\hline 15 & $\mathrm{~F}$ & 0.584 & 0.010 & 0.379 & 0.0147 & 57.7 & 5.77 & 4.5 & 0.58 \\
\hline 16 & $\mathrm{G}$ & 1.500 & 0.017 & 0.346 & 0.0073 & 57.4 & 3.38 & 1.5 & 0.33 \\
\hline 17 & $\mathrm{H}$ & 1.798 & 0.020 & 0.296 & 0.0071 & 43.7 & 4.68 & -3.3 & 0.29 \\
\hline 18 & $\mathrm{H}$ & 2.480 & 0.024 & 0.253 & 0.0060 & 75.1 & 2.50 & -0.4 & 0.22 \\
\hline 19 & I & 1.140 & 0.014 & 0.296 & 0.0100 & 72.8 & 3.60 & 5.1 & 0.34 \\
\hline 20 & I & 1.879 & 0.020 & 0.356 & 0.0062 & 59.2 & 2.83 & -1.5 & 0.30 \\
\hline 21 & I & 3.389 & 0.031 & 0.260 & 0.0049 & 49.6 & 3.29 & -6.5 & 0.21 \\
\hline 22 & $\mathrm{~J}$ & 2.151 & 0.013 & 0.312 & 0.0027 & 55.6 & 1.60 & -1.6 & 0.15 \\
\hline 23 & $\mathrm{~K}$ & 2.900 & 0.027 & 0.249 & 0.0054 & 75.5 & 2.32 & -5.0 & 0.21 \\
\hline 24 & $\mathrm{~L}$ & 2.880 & 0.027 & 0.300 & 0.0050 & 67.6 & 2.26 & 8.2 & 0.23 \\
\hline 25 & $\mathrm{~L}$ & 2.515 & 0.025 & 0.342 & 0.0053 & 41.3 & 3.71 & -14.1 & 0.27 \\
\hline 26 & M & 3.587 & 0.021 & 0.237 & 0.0039 & 61.7 & 2.06 & 1.4 & 0.14 \\
\hline 27 & M & 3.750 & 0.034 & 0.258 & 0.0045 & 60.2 & 2.56 & -2.2 & 0.20 \\
\hline 28 & $\mathrm{~N}$ & 3.457 & 0.034 & 0.461 & 0.0044 & 46.0 & 2.43 & 0.8 & 0.30 \\
\hline 29 & $\mathrm{O}$ & 3.690 & 0.035 & 0.407 & 0.0042 & 52.6 & 2.20 & 0.4 & 0.26 \\
\hline 30 & $\mathrm{O}$ & 3.420 & 0.032 & 0.459 & 0.0042 & 74.1 & 1.46 & -3.8 & 0.26 \\
\hline 31 & $\mathrm{O}$ & 2.150 & 0.022 & 0.436 & 0.0052 & 87.5 & 1.52 & 3.7 & 0.27 \\
\hline 32 & $\mathrm{O}$ & 5.012 & 0.037 & 0.415 & 0.0035 & 0.0 & 4.30 & -3.1 & 0.26 \\
\hline 33 & $\mathrm{O}$ & 2.819 & 0.028 & 0.629 & 0.0049 & 75.2 & 1.32 & -3.9 & 0.36 \\
\hline 34 & $\mathrm{O}$ & 3.895 & 0.036 & 0.327 & 0.0042 & 34.5 & 3.84 & -0.2 & 0.23 \\
\hline 35 & $\mathrm{O}$ & 2.970 & 0.030 & 0.727 & 0.0052 & 75.2 & 1.23 & -1.1 & 0.40 \\
\hline 36 & $\mathrm{O}$ & 3.599 & 0.036 & 0.756 & 0.0052 & 72.3 & 1.22 & -1.9 & 0.42 \\
\hline 37 & $\mathrm{O}$ & 3.090 & 0.032 & 0.901 & 0.0060 & 75.0 & 1.15 & -0.8 & 0.46 \\
\hline 38 & $\mathrm{O}$ & 3.672 & 0.037 & 0.902 & 0.0058 & 75.5 & 1.11 & -1.9 & 0.44 \\
\hline 39 & $\mathrm{O}$ & 3.371 & 0.032 & 0.646 & 0.0045 & 92.5 & 0.98 & -7.4 & 0.27 \\
\hline 40 & $\mathrm{O}$ & 3.810 & 0.038 & 0.792 & 0.0053 & 72.6 & 1.18 & 4.6 & 0.43 \\
\hline 41 & $\mathrm{O}$ & 3.230 & 0.033 & 0.939 & 0.0062 & 73.9 & 1.15 & 132.3 & 0.47 \\
\hline 42 & $\mathrm{P}$ & 4.539 & 0.041 & 0.369 & 0.0038 & 48.9 & 2.34 & 5.0 & 0.23 \\
\hline 43 & $\mathrm{Q}$ & 2.740 & 0.030 & 0.916 & 0.0064 & 64.5 & 1.41 & 89.5 & 0.63 \\
\hline 44 & $\mathrm{R}$ & 7.501 & 0.065 & 0.638 & 0.0039 & 82.8 & 0.94 & 92.3 & 0.27 \\
\hline 45 & $\mathrm{R}$ & 6.998 & 0.064 & 0.735 & 0.0046 & 65.9 & 1.23 & 96.4 & 0.41 \\
\hline 46 & $\mathrm{R}$ & 7.513 & & 0.741 & & 79.4 & & 96.9 & \\
\hline 47 & $\mathrm{R}$ & 9.055 & & 0.653 & & 73.7 & & 98.6 & \\
\hline 48 & $\mathrm{R}$ & 6.238 & & 0.854 & & 53.8 & & 106.9 & \\
\hline 49 & $\mathrm{R}$ & 11.519 & 0.110 & 0.802 & 0.0051 & 22.7 & 3.66 & 104.0 & 0.82 \\
\hline 50 & $\mathrm{R}$ & 10.901 & 0.090 & 0.486 & 0.0032 & 73.5 & 1.10 & 96.2 & 0.23 \\
\hline
\end{tabular}




\begin{tabular}{rrrrrrrrrr}
\multicolumn{1}{c}{ continued from previous page } \\
\hline $\begin{array}{r}\text { Insect } \\
\text { number }\end{array}$ & $\begin{array}{r}\text { Species } \\
\text { symbol }\end{array}$ & $\begin{array}{r}\sigma_{x x} \\
\mathrm{~cm}^{2}\end{array}$ & $\begin{array}{r}\delta \sigma_{x x} \\
\mathrm{~cm}^{2}\end{array}$ & $\epsilon$ & $\delta \epsilon$ & $\begin{array}{r}\beta \\
\circ\end{array}$ & $\begin{array}{r}\delta \beta \\
{ }^{\circ}\end{array}$ & $\begin{array}{r}\theta_{0} \\
{ }^{\circ}\end{array}$ & $\begin{array}{r}\delta \theta_{0} \\
\circ\end{array}$ \\
\hline 51 & $\mathrm{R}$ & 15.210 & 0.134 & 0.627 & 0.0039 & 44.9 & 1.80 & 90.5 & 0.37 \\
52 & $\mathrm{R}$ & 17.053 & 0.150 & 0.644 & 0.0040 & 48.7 & 1.63 & 101.5 & 0.38 \\
53 & $\mathrm{R}$ & 15.330 & 0.121 & 0.401 & 0.0027 & 71.6 & 1.14 & 88.6 & 0.19 \\
54 & $\mathrm{R}$ & 32.472 & 0.260 & 0.479 & 0.0029 & 62.2 & 1.23 & 91.2 & 0.22 \\
\hline
\end{tabular}

\begin{tabular}{|c|c|c|c|c|c|}
\hline $\begin{array}{r}\text { Insect } \\
\text { number }\end{array}$ & $\begin{array}{l}\text { Species } \\
\text { symbol }\end{array}$ & Species & Mass & $\begin{array}{r}\text { Body } \\
\text { length } \\
\mathrm{mm}\end{array}$ & $\begin{array}{r}\text { Abdomen } \\
\text { width } \\
\text { mm }\end{array}$ \\
\hline 1 & $\mathrm{~S}$ & Heliothis zea (female) & 254 & 17.0 & 5.0 \\
\hline 2 & $\mathrm{~S}$ & Heliothis zea (male) & 206 & 14.5 & 3.5 \\
\hline 3 & $\mathrm{~T}$ & Honeybee (drone) & 182 & 14.0 & 5.0 \\
\hline 4 & $\mathrm{~T}$ & Honeybee (drone) & 208 & 14.5 & 5.5 \\
\hline 5 & $\mathrm{~T}$ & Honeybee (queen) & 185 & 16.0 & 4.5 \\
\hline 6 & $\mathrm{~T}$ & Honeybee (queen) & 196 & 16.0 & 4.5 \\
\hline 7 & $\mathrm{~T}$ & Honeybee (worker) & 150 & 11.0 & 4.0 \\
\hline 8 & $\mathrm{~T}$ & Honeybee (worker) & 99 & 11.0 & 4.0 \\
\hline 9 & $\mathrm{U}$ & Stink Bug & 132 & 14.5 & 8.0 \\
\hline 10 & $\mathrm{~V}$ & Fall Armyworm & 100 & 14.5 & 3.5 \\
\hline 11 & W & L.c.s. borer & 14 & 10.0 & 2.0 \\
\hline 12 & $\mathrm{~W}$ & L.c.s. borer & 9 & 8.0 & 1.4 \\
\hline 13 & $\mathrm{X}$ & Boll Weevil & 10 & 5.0 & 2.0 \\
\hline 14 & $\mathrm{X}$ & Boll Weevil & 11 & 5.0 & 2.0 \\
\hline
\end{tabular}

Table 4:

Physical data and species of measured insects of [9].

\section{Discussion and Conclusions}

The results provide a valuable reference for work in radar remote sensing, especially radar entomology. Both models used (harmonic and SM3) have their strengths and should be used appropriately. Although care is required to quantify the measurement uncertainty, the added value of having quantitative parameter uncertainties when using the maximum likelihood methodology is a significant benefit, and in a fundamental sense is a necessary step for reliable interpretation of the fitted parameters.

The measurement methods used give good results, even with relatively simple equipment. In passing we note the ability of a co-polar measurement method [10] to obtain information about the cross-polar scattering terms - although an ambiguity cannot be resolved to give a complete measurement.

The results presented here usefully augment the insect RCS data in the formal open literature, and use parameterizations suitable for a wide range of applications. An area of potential ambiguity in practice is that the apparent orientation is ambiguous by $90^{\circ}$ with respect to swapping the $x$ and $y$ axes. 


\begin{tabular}{rrrrrrrrrrr}
$\begin{array}{r}\text { Insect } \\
\text { number }\end{array}$ & $\begin{array}{r}\text { Species } \\
\text { symbol }\end{array}$ & $\begin{array}{r}a_{0} \\
\mathrm{~cm}^{2}\end{array}$ & $\begin{array}{r}a_{1} \\
\mathrm{~cm}^{2}\end{array}$ & $\begin{array}{r}a_{2} \\
\mathrm{~cm}^{2}\end{array}$ & $\begin{array}{r}\theta_{1} \\
{ }^{\circ}\end{array}$ & $\begin{array}{r}\theta_{2}-\theta_{1} \\
{ }^{\circ}\end{array}$ & $\begin{array}{r}\sigma_{x x} \\
\mathrm{~cm}^{2}\end{array}$ & $\epsilon$ & $\begin{array}{r}\beta \\
\circ\end{array}$ & $\begin{array}{r}\theta_{0} \\
{ }^{\circ}\end{array}$ \\
\hline 1 & $\mathrm{~S}$ & 1.790 & 1.620 & 0.291 & -1.2 & 2.7 & 3.697 & 0.352 & 44.4 & -0.9 \\
2 & $\mathrm{~S}$ & 0.410 & 0.390 & 0.071 & 0.9 & -1.9 & 0.871 & 0.323 & 45.1 & 0.7 \\
3 & $\mathrm{~T}$ & 0.780 & 0.540 & 0.243 & 0.1 & -0.3 & 1.563 & 0.556 & 86.6 & -0.0 \\
4 & $\mathrm{~T}$ & 1.040 & 0.640 & 0.285 & 0.9 & -1.4 & 1.964 & 0.590 & 80.8 & 0.3 \\
5 & $\mathrm{~T}$ & 1.030 & 1.060 & 0.495 & -0.6 & 0.9 & 2.585 & 0.424 & 114.5 & -0.2 \\
6 & $\mathrm{~T}$ & 0.820 & 0.880 & 0.328 & -0.5 & 1.0 & 2.028 & 0.363 & 102.8 & -0.1 \\
7 & $\mathrm{~T}$ & 0.150 & 0.140 & 0.023 & -0.7 & 1.7 & 0.313 & 0.325 & 36.8 & -0.5 \\
8 & $\mathrm{~T}$ & 0.410 & 0.380 & 0.049 & 0.7 & -1.9 & 0.840 & 0.310 & 0.0 & 0.6 \\
9 & $\mathrm{U}$ & 1.480 & 0.980 & 0.175 & 1.0 & -2.3 & 2.633 & 0.506 & 43.8 & 0.7 \\
10 & $\mathrm{~V}$ & 0.530 & 0.600 & 0.149 & 1.5 & -3.0 & 1.277 & 0.245 & 73.4 & 0.9 \\
11 & $\mathrm{~W}$ & 0.081 & 0.087 & 0.015 & 1.6 & -0.7 & 0.183 & 0.222 & 27.4 & 1.5 \\
12 & $\mathrm{~W}$ & 0.013 & 0.013 & 0.003 & -1.4 & 2.8 & 0.029 & 0.353 & 75.1 & -0.8 \\
13 & $\mathrm{X}$ & 0.005 & 0.004 & 0.001 & -0.8 & 2.2 & 0.010 & 0.326 & 7.1 & -0.6 \\
14 & $\mathrm{X}$ & 0.013 & 0.010 & 0.001 & -0.5 & 2.2 & 0.024 & 0.394 & 0.0 & -0.4
\end{tabular}

Table 5: Harmonic and SM3 parameters for insects of [9]. The harmonic parameters are those measured by Wolf et al.; the SM3 parameters are new results presented here.

The data here avoid this since the true orientations of the measured insects are known.

Areas of further work which would usefully build on these results are (1) publication of more measurements of insect RCS to extend the range of species represented, (2) modelling (e.g. of water ellipsoids) to help interpret these measurements, and (3) development of algorithms to determine the extent to which RCS parameters can be used to identify insects (noting recent work [13] which adds wingbeat frequency to the range of measurable parameters).

\section{Acknowledgements}

The experiments would not have been possible without the support and loan of equipment by Prof. J.R. Riley and Mr. A. Smith (Rothamsted Research, UK). The assistance of Dr. S.H. Al-Charchafchi (Cranfield University) with the microwave receiver calibration was also much appreciated. The anonymous reviewers' constructive criticism has been helpful and is appreciated.

\section{References}

[1] T.J. Dean and V.A. Drake. Monitoring insect migration with radar: the ventral-aspect polarization pattern and its potential for target identification. International Journal of Remote Sensing, 26(18):3957-3974, September 2005 .

[2] J.W. Chapman, D.R. Reynolds, and A.D. Smith. Vertical-looking radar: a new tool for monitoring high-altitude insect migration. BioScience, 53(5):503-511, May 2003. 
[3] A.D. Smith, D.R. Reynolds, and J.R. Riley. The use of vertical-looking radar to continuously monitor the insect fauna flying at altitude over southern England. Bulletin of Entomological Research, 90:265-277, 2000.

[4] J.R. Riley, A.D. Smith, and D.R. Reynolds. The feasibility of using verticallooking radar to monitor the migration of brown planthopper and other insect pests of rice in china. Entomologia Sinica, 10(1):1-19, March 2003.

[5] A.D. Smith, J.R. Riley, and R.D. Gregory. A method for routine monitoring of the aerial migration of insects by using a vertical-looking radar. Phil. Trans. R. Soc. Lond. B, 340:393-404, 1993.

[6] Stephen E. Hobbs, K. Allsopp, and W. Wolf. Signal analysis for an entomological radar with a vertical nutating beam. College of Aeronautics report 9919, Cranfield University, March 2000. ISBN 186194 0483, doi http://hdl.handle.net/1826/73.

[7] I.T. Harman and V.A. Drake. Insect monitoring radar: analytical timedomain algorithm for retrieving trajectory and target parameters. Computers and Electronics in Agriculture, 43:23-41, 2004.

[8] J.R. Riley. Radar cross section of insects. Proc. IEEE, 73(2):228-232, 1985.

[9] W.W. Wolf, C.R. Vaughn, R. Harris, and G.M. Loper. Insect radar crosssections for aerial density measurements and target classification. Transactions of the American Society of Agricultural Engineers, 36(3):949-954, 1993.

[10] A.C. Aldhous. An investigation of the polarisation dependence of insect radar cross sections at constant aspect. PhD thesis, Ecological Physics Research Group, Cranfield Institute of Technology, 1989.

[11] William H. Press, Saul A. Teukolsky, William T. Vetterling, and Brian P. Flannery. Numerical recipes in C. Cambridge University Press, second edition, 1992.

[12] J.W. Chapman, A.D. Smith, I.P. Woiwood, D.R. Reynolds, and J.R. Riley. Development of vertical-looking radar technology for monitoring insect migration. Computers and Electronics in Agriculture, 35:95-110, 2002.

[13] H.K. Wang and V.A. Drake. Insect monitoring radar: retrieval of wingbeat information from conical-scan observation data. Computers and Electronics in Agriculture, 43:209-222, 2004. 